\title{
INTEGRATION OPERATOR ACTING ON HARDY AND WEIGHTED BERGMAN SPACES
}

\author{
JOUNI RÄTTYÄ
}

Questions related to the operator $J_{g}(f)(z):=\int_{0}^{z} f(\zeta) g^{\prime}(\zeta) d \zeta$, induced by an analytic function $g$ in the unit disc, are studied. It is shown that a function $G$ is the derivative of a function in the Hardy space $H^{p}$ if and only if it is of the form $G=F \psi^{\prime}$ where $F \in H^{q}, \psi \in H^{s}$ and $1 / s=1 / p-1 / q$. Moreover, a complete characterisation of when $J_{g}$ is bounded or compact from one weighted Bergman space $A_{\alpha}^{p}$ into another is established, and an asymptotic formula for the essential norm of $J_{g}$, the distance from compact operators in the operator norm, is given. As an immediate consequence it is obtained that if $p<2+\alpha$ and $\alpha>-1$, then any primitive of $f \in A_{\alpha}^{p}$ belongs to $A_{\alpha}^{q}$ where $q=((2+\alpha) p) /(2+\alpha-p)$. For $\alpha=-1$ this is a sharp result by Hardy and Littlewood on primitives of functions in Hardy space $H^{p}=A_{-1}^{p}, 0<p<1$.

\section{INTRODUCTION AND MAIN RESULTS}

Every analytic function $g$ in the open unit disc $\mathbb{D}$ of the complex plane induces the linear integration operator

$$
J_{g}(f)(z):=\int_{0}^{z} f(\zeta) g^{\prime}(\zeta) d \zeta
$$

acting on the space $H(\mathbb{D})$ of all analytic functions in $\mathbb{D}$. If $g(z)=z$ or $g(z)=-\log (1-z)$, then $J_{g}$ is the integration operator or the Cesáro operator, respectively. The operator $J_{g}$ has been studied, for instance, in $[1,2,3,20,23]$. Further, the operator $J_{g}$ arises naturally in the study of semigroups of composition operators acting on spaces of analytic functions [22].

In this paper questions related to the operator $J_{g}$ and Hardy and weighted Bergman spaces are studied. A representation for derivatives of $H^{p}$-functions, Theorem 1 , is first derived from results, related to the operator $J_{g}$, in the existing literature. Then, in the same spirit as in the theory of composition operators [21], the essential norm of $J_{g}$ mapping from one Banach space of analytic functions in $\mathbf{D}$ into another is defined

Received 6th November, 2006

This research was supported in part by the MEC-Spain, MTM2005-07347, the Spanish Thematic Network MTM2006-26627-E, and the Academy of Finland 210245.

Copyright Clearance Centre, Inc. Serial-fee code: 0004-9727/07 \$A2.00+0.00. 
as the distance of $J_{g}$ from compact operators in the operator norm. An asymptotic formula for the essential norm of $J_{g}$ acting on the Hardy space $H^{2}$ is given in Theorem 1 . A complete characterisation of when $J_{g}$ is a bounded operator between two weighted Bergman spaces is established in Theorem 2. This yields Corollary 3 and its strengthened version, Theorem 4, of which the first one can be understood as a generalisation of a sharp result by Hardy and Littlewood on primitives of $H^{p}$-functions. Theorem 5 gives a characterisation for $J_{g}$ to be bounded from the Hardy space $H^{p}$ into the weighted Bergman space $A_{\beta}^{q}$ when $p \leqslant q$. An asymptotic formula for the essential norm of $J_{g}$ acting from one weighted Bergman space into another is given in Theorem 6 . This yields a characterisation, Corollary 7 , of when $J_{g}$ acts as a compact operator between two weighted Bergman spaces. Finally, two of observations on exponentials of $B M O A$ - and Bloch-functions are made, see the discussion related to Proposition 8.

Before continuing further, one more observation is made. Namely, if the target space is the weighted Bergman space $A_{\beta}^{q}, \beta>-1$, then the operator $J_{g}$ from $A_{\alpha}^{p}, \alpha \geqslant-1$, to $A_{\beta}^{q}$ is bounded if and only if the multiplication operator $M_{g^{\prime}}$, defined by $M_{g^{\prime}}(f)(z)$ $:=f(z) g^{\prime}(z)$, is bounded from $A_{\alpha}^{p}$ to $A_{q+\beta}^{q}$. Therefore Theorems 2 and 5 can also be deduced by Zhao's results on multiplication operators [29]. However, since the aim of the present paper is slightly different and also for convenience of the reader, the proofs of these results are presented in the setting of the operator $J_{g}$.

Pommerenke [20] showed that $J_{g}$ is bounded on the Hardy space $H^{2}$ if and only if $g$ belongs to $B M O A$, the space of analytic functions of bounded mean oscillation. This was extended to other Hardy spaces by Aleman, Cima and Siskakis $[1,2]: J_{g}$ is bounded on $H^{p}, 0<p<\infty$, if and only if $g$ belongs to $B M O A$. It is worth noticing that $g \in B M O A$ if and only if $\sup _{a \in \mathbb{D}}\left\|g \circ \varphi_{a}-g(a)\right\|_{H^{p}}<\infty$, where $\varphi_{a}$ is the automorphism of $\mathbb{D}$ which interchanges zero and the point $a \in \mathbb{D},[5]$. For $p>0$, the Hardy space $H^{p}$ consists of those functions $f \in H(\mathbb{D})$ that satisfy

$$
\|f\|_{H^{p}}^{p}:=\lim _{r \rightarrow 1^{-}} \frac{1}{2 \pi} \int_{0}^{2 \pi}\left|f\left(r e^{i \theta}\right)\right|^{p} d \theta<\infty .
$$

Moreover, Aleman and Cima [1] gave a complete characterisation of bounded and compact operators $J_{g}: H^{p} \rightarrow H^{q}$ in terms of the symbol $g$. Furthermore, Siskakis and Zhao [23] found a necessary and sufficient condition for $g$ such that $J_{g}$ is bounded or compact on $B M O A$.

A result by Cohn on factorisation of derivatives of $H^{p}$-functions is related to the operator $J_{g}$ acting on $H^{p}$. Cohn [9, Theorem 1] showed that a function $G$ is the derivative of an $H^{p}$-function if and only if it can be represented in the form $G=F \psi^{\prime}$ where $F \in H^{p}$ and $\psi \in B M O A$. This clearly implies that $J_{g}$ is bounded on $H^{p}$ if $g \in B M O A$, and improves [1, Lemma 2] which states that $G$ must be of the form $G=\sum_{j=1}^{4} F_{j} \psi_{j}^{\prime}$ where $F_{j} \in H^{p}$ and $\psi_{j} \in B M O A$ for all $j=1,2,3,4$. The result by Cohn together with the 
characterisation of Aleman and Cima of when $J_{g}: H^{p} \rightarrow H^{q}, q<p$, is bounded yield the following representation of derivatives of $H^{p}$-functions.

THEDREM A. Let $0<p<q<\infty$. A function $G$ is the derivative of a function in $H^{p}$ if and only if it can be represented in the form $G=F \psi^{\prime}$ where $F \in H^{q}, \psi \in H^{s}$ and $1 / s=1 / p-1 / q$.

If $G$ is of the form $G=F \psi^{\prime}$, where $F \in H^{q}$ and $\psi \in H^{s}$ such that $1 / s=1 / p-1 / q$, then any primitive of $G$ belongs to $H^{p}$ since the operator $J_{\psi}: H^{q} \rightarrow H^{p}, p<q$, is bounded by the result of Aleman and Cima [1, Theorem 1(i)].

Conversely, if $p<q$ and $f \in H^{p}$, then $f^{\prime}$ can be represented in the form $f^{\prime}=F_{1} \varphi^{\prime}$ where $F_{1} \in H^{p}$ and $\varphi \in B M O A$ by the result of Cohn. Further, by the general factorisation of $H^{p_{\text {-functions }}} F_{1}$ can be represented in the form $F_{1}=F F_{2}$, where $F \in H^{q}, F_{2} \in H^{s}$ and $1 / s=1 / p-1 / q$, so that $f^{\prime}=F F_{2} \varphi^{\prime}$. But $F_{2} \varphi^{\prime}$ must be the derivative of a function in $H^{s}$ by the result of Cohn, say $\psi^{\prime}=F_{2} \varphi^{\prime}$, where $\psi \in H^{s}$. Thus $f^{\prime}=F \psi^{\prime}$ where $F \in H^{q}$ and $\psi \in H^{s}$ such that $1 / s=1 / p-1 / q$ as desired.

For two Banach spaces $X, Y \subset H(\mathbb{D})$, the essential norm $\left\|J_{g}\right\|_{e}$ of the bounded operator $J_{g}: X \rightarrow Y$ is defined as the distance of $J_{g}$ from compact operators in the operator norm, that is, $\left\|J_{g}\right\|_{e}:=\inf _{K}\left\|J_{g}-K\right\|$ where the infimum is taken over all compact operators $K: X \rightarrow Y$.

The following resuit gives an asymptotic formula for the essential norm of $J_{g}$ acting on $H^{2}$, and yields the known fact that $J_{g}$ is compact on $H^{2}$ if and only if $g$ belongs to $V M O A$, the space of analytic functions of vanishing mean oscillation [1]. Its proof, as well as other rather lengthy and technical proofs are postponed to Section 3 . Here and hereafter $d A$ denotes the Lebesgue area element normalised such that $A(\mathbb{D})=1$.

THEOREM 1. If $J_{g}$ is bounded on $H^{2}$, then there is a positive constant $C$ such that

(1.1) $\limsup _{|a| \rightarrow 1^{-}} \int_{\mathbb{D}}\left|g^{\prime}(z)\right|^{2}\left(1-\left|\varphi_{a}(z)\right|^{2}\right) d A(z)$

$$
\leqslant\left\|J_{g}\right\|_{e}^{2} \leqslant C \limsup _{|a| \rightarrow 1^{-}} \int_{\mathbb{D}}\left|g^{\prime}(z)\right|^{2}\left(1-\left|\varphi_{a}(z)\right|^{2}\right) d A(z) .
$$

A generalisation of Theorem 1 for Dirichlet type space $D^{p}, 0<p \leqslant 2$, consisting of those $f \in H(\mathbb{D})$ for which the integral $\int_{\mathbb{D}}\left|f^{\prime}\right|^{p}\left(1-|z|^{2}\right)^{p-1} d A$ converges, is established in Section 3, where a characterisation of when $J_{g}$ is bounded on $D^{p}$ is also given.

Aleman and Siskakis [3] showed that $J_{g}$ is bounded on the weighted Bergman space $A_{\alpha}^{p}, 0<p<\infty$ and $-1<\alpha<\infty$, if and only if $g$ belongs to the Bloch space $\mathcal{B}$, that is, $J_{g}$ is bounded on $A_{\alpha}^{p}$ if and only if $\sup _{a \in D}\left\|g \circ \varphi_{a}-g(a)\right\|_{A_{a}^{p}}<\infty$. They also considered weighted Bergman spaces defined by using certain more general weights and characterised the compact operators $J_{g}$ on $A_{\alpha}^{p}: J_{g}$ is compact on $A_{\alpha}^{p}$ if and only if $g$ belongs to the 
little Bloch space $\mathcal{B}_{0}$. For $0<p<\infty$ and $-1<\alpha<\infty$, the weighted Bergman space $A_{\alpha}^{p}$ consists of those functions $f \in H(\mathbb{D})$ for which

$$
\|f\|_{A_{a}^{p}}:=\left((\alpha+1) \int_{\mathbf{D}}|f(z)|^{p}\left(1-|z|^{2}\right)^{\alpha} d A(z)\right)^{1 / p}<\infty .
$$

As usual, writing $A_{-1}^{p}:=H^{p}$, the space $H^{p}$ is identified with the limit space of the weighted Bergman space $A_{\alpha}^{p}$ as $\alpha \rightarrow-1^{+}$[28]. Further, a function $f \in H(\mathbb{D})$ belongs to the $\alpha$-Bloch space $\mathcal{B}^{\alpha}, 0 \leqslant \alpha<\infty$, if

$$
\|f\|_{B^{\alpha}}:=\sup _{z \in \mathbf{D}}\left|f^{\prime}(z)\right|\left(1-|z|^{2}\right)^{\alpha}<\infty .
$$

The little $\alpha$-Bloch space $\mathcal{B}_{0}^{\alpha}, 0<\alpha<\infty$, consists of those $f \in H(\mathbb{D})$ for which $\left|f^{\prime}(z)\right|(1$ $\left.-|z|^{2}\right)^{\alpha} \rightarrow 0$, as $|z| \rightarrow 1$. The standard Bloch and little Bloch spaces are $\mathcal{B}^{1}:=\mathcal{B}$ and $\mathcal{B}_{0}^{1}:=\mathcal{B}_{0}$. It is known that $B^{\alpha}$ is the analytic Lipschitz class $\Lambda_{1-\alpha}$ for $0 \leqslant \alpha<1$. A similar result holds for the corresponding little spaces.

Theorem 2 gives a complete characterisation of bounded operators $J_{g}$ mapping from the weighted Bergman space $A_{\alpha}^{p}$ into another weighted Bergman space $A_{B}^{q}$ in terms of the symbol $g$. The case when $J_{g}$ acts on $A_{a}^{p}$ is due to Aleman and Siskakis [3].

THEOREM 2. Let $0<p, q<\infty$ and $-1<\alpha, \beta<\infty$.

(i) If $p \leqslant q$ and $(2+\alpha) / p-(2+\beta) / q \leqslant 1$, then $J_{g}: A_{\alpha}^{p} \rightarrow A_{\beta}^{q}$ is bounded if and only if $g \in \mathcal{B}^{1+(2+\beta) / q-(2+\alpha) / p}$.

(ii) If $q<p$ and $(2+\alpha) / p-(2+\beta) / q \leqslant 1$. Then $J_{g}: A_{\alpha}^{p} \rightarrow A_{\beta}^{q}$ is bounded if and only if $g^{\prime} \in A_{s+\gamma}^{s}$, where $1 / s=1 / q-1 / p$ and $\gamma=(p \beta-\alpha q) /(p-q)$. In particular, $J_{g}: A_{\alpha}^{p} \rightarrow A_{\alpha}^{q}$ is bounded if and only if $g \in A_{\alpha}^{s}$, where $1 / s=1 / q-1 / p$.

(iii) If $(2+\alpha) / p-(2+\beta) / q>1$ and $J_{g}: A_{\alpha}^{p} \rightarrow A_{\beta}^{q}$ is bounded, then $g$ is constant.

If $g(z)=z, \alpha=\beta$ and $1 / p-1 / q=1 /(2+\alpha)$ in Theorem 2(i), Corollary 3 is obtained. Taking in account the identification $H^{p}=A_{-1}^{p}$, it can be understood as a generalisation of the sharp result by Hardy and Littlewood which states that any primitive function of $f \in H^{p}, 0<p<1$, belongs to $H^{q}$ where $q=p /(1-p)$, see [12].

Corollary 3. Let $0<p<2+\alpha$ and $-1<\alpha<\infty$. Then any primitive of $f \in A_{\alpha}^{p}$ belongs to $A_{\alpha}^{q}$ where $q=((2+\alpha) p) /(2+\alpha-p)$.

The result of Hardy and Littlewood was in a sense improved in [1]. In a similar manner Corollary 3 has the following strengthened version.

THEOREM 4. Let $0<p<2+\alpha,-1<\alpha<\infty, 0<p_{1}<\infty$ and $2+\alpha<p_{2}<\infty$ such that $1 / p=1 / p_{1}+1 / p_{2}$. If $f$ is analytic in $D$ with a representation of the form $f=f_{1} f_{2}$, where $f_{1} \in A_{\alpha}^{p_{1}}$ and $f_{2} \in \mathcal{B}^{1+(2+\alpha) / p_{2}}$, then any primitive of $f$ belongs to $A_{\alpha}^{q}$ where $q=((2+\alpha) p) /(2+\alpha-p)$. 
By the assumption, any primitive $F_{2}$ of $f_{2}$ belongs to $\mathcal{B}^{(\alpha+2) / p_{2}}$. Therefore, by Theorem 2(i), the operator $J_{F_{2}}$ maps $A_{\alpha}^{p_{1}}$ into $A_{\alpha}^{q}$ where $1+(2+\alpha) / q-(2+\alpha) / p_{1}=(2+\alpha) / p_{2}$, that is, $q=((2+\alpha) p) /(2+\alpha-p)$. But $J_{F_{2}}\left(f_{1}\right)$ is a primitive of $f$, and so it belongs to $A_{\alpha}^{q}$ where $q=((2+\alpha) p) /(2+\alpha-p)$.

In the case when $p \leqslant q$ and $\alpha=-1$ the following result is offered.

THEOREM 5. Let $0<p, q<\infty$ and $-1<\beta<\infty$.

(i) If $p<q$ and $1 / p-(2+\beta) / q \leqslant 1$, then $J_{g}: H^{p} \rightarrow A_{\beta}^{q}$ is bounded if and only if $g \in \mathcal{B}^{1+(2+\beta) / q-1 / p}$.

(ii) If $\beta \leqslant p+1$. Then $J_{g}: H^{p} \rightarrow A_{\beta}^{p}$ is bounded if and only if

$$
\sup _{a \in \mathbb{D}} \int_{\mathbb{D}}\left|g^{\prime}(z)\right|^{p}\left(1-|z|^{2}\right)^{p+\beta-1}\left(1-\left|\varphi_{a}(z)\right|^{2}\right) d A(z)<\infty .
$$

(iii) If $1 / p-(2+\beta) / q>1$ and $J_{g}: H^{p} \rightarrow A_{\beta}^{q}$ is bounded, then $g$ is constant.

An asymptotic formula for the essential norm of $J_{g}$ acting from one weighted Bergman space into another is given in Theorem 6. The symbol $\simeq$ in the statement means that the quantities in other sides of the symbol are comparable, that is, their quotient is bounded and bounded away from zero.

TheOrem 6. Let $1<p, q<\infty$ and $-1 \leqslant \alpha, \beta<\infty$ such that $(2+\alpha) / p$ $-(2+\beta) / q \leqslant 1$, and let $J_{g}: A_{\alpha}^{p} \rightarrow A_{\beta}^{q}$ be bounded.

(i) If $p \leqslant q$ and $-1<\alpha, \beta<\infty$, then

$$
\left\|J_{g}\right\|_{e} \simeq \limsup _{|z| \rightarrow 1^{-}}\left|g^{\prime}(z)\right|\left(1-|z|^{2}\right)^{1+(2+\beta) / q-(2+\alpha) / p} .
$$

(ii) If $p<q, \alpha=-1$ and $-1<\beta<\infty$, then

$$
\left\|J_{g}\right\|_{e} \simeq \limsup _{|z| \rightarrow 1^{-}}\left|g^{\prime}(z)\right|\left(1-|z|^{2}\right)^{1+(2+\beta) / q-1 / p} .
$$

(iii) If $q=p$ and $-1<\beta \leqslant p+1$, then

$$
\left\|J_{g}\right\|_{e}^{p} \simeq \limsup _{|a| \rightarrow 1^{-}} \int_{\mathbb{D}}\left|g^{\prime}(z)\right|^{p}\left(1-|z|^{2}\right)^{p+\beta-1}\left(1-\left|\varphi_{a}(z)\right|^{2}\right) d A(z) .
$$

Theorem 6 yields the following characterisation of compact operators $J_{g}: A_{\alpha}^{p} \rightarrow A_{\beta}^{q}$.

Corollary 7. Let $1<p, q<\infty$ and $-1<\alpha, \beta<\infty$ such that $(2+\alpha) / p$ $-(2+\beta) / q \leqslant 1$.

(i) If $p \leqslant q$, then $J_{g}: A_{\alpha}^{p} \rightarrow A_{\beta}^{q}$ is compact if and only if $g \in \mathcal{B}_{0}^{1+(2+\beta) / q-(2+\alpha) / p}$.

(ii) If $p<q$, then $J_{g}: H^{p} \rightarrow A_{\beta}^{q}$ is compact if and only if $g \in \mathcal{B}_{0}^{1+(2+\beta) / q-1 / p}$.

(iii) If $\beta \leqslant p+1$, then $J_{g}: H^{p} \rightarrow A_{\beta}^{p}$ is compact if and only if

$$
\lim _{|a| \rightarrow 1} \int_{\mathbf{D}}\left|g^{\prime}(z)\right|^{p}\left(1-|z|^{2}\right)^{p+\beta-1}\left(1-\left|\varphi_{a}(z)\right|^{2}\right) d A(z)=0 .
$$


Finally, the exponentials of $B M O A-$ and Bloch-functions are shortly discussed. Cima and Schober [8] showed that for any function $f$ in $B M O A$ there is a $p$ such that $e^{f}$ belongs to $H^{p}$. This result has the following easy analogue.

Proposition 8. For any $f \in \mathcal{B}$ and $-1<\alpha<\infty$ there is a $p>0$ such that $e^{f}$ belongs to $A_{\alpha}^{p}$. Symbolically, $\exp \mathcal{B} \subset \bigcup_{p>0} A_{\alpha}^{p}$.

Since $|f(z)| \leqslant\|f\|_{B} \log 2 /(1-|z|)+|f(0)|$ it follows that

$$
\left\|e^{f}\right\|_{A_{\alpha}^{p}}^{p} \leqslant e^{p|f(0)|} 2^{p\|f\|_{\mathcal{B}}} \int_{\mathbb{D}} \frac{d A(z)}{\left(1-|z|^{2}\right)^{p\|f\|_{\mathcal{B}-\alpha}}}
$$

so if $p<(1+\alpha) /\|f\|_{\mathcal{B}}$, then $e^{f}$ belongs to $A_{\alpha}^{p}$ as desired.

Proposition 8 and the result of Cima and Schober are sharp in the sense that there is a function $f$ in the little Bloch space $\mathcal{B}_{0}$ such that $e^{f}$ does not belong to $\bigcup_{p>0} H^{p}$. To see this, let $f(z):=\sum_{k=1}^{\infty} a_{k} z^{n_{k}}=\sum_{k=1}^{\infty} k^{-(1 / 2)} z^{k^{k}}$. Then $f$ is analytic in $\mathbb{D}$ and it has Hadamard gaps; $\left(n_{k+1}\right) / n_{k} \geqslant 2$ for all $k \in \mathbb{N}$. Further, since $\limsup _{k \rightarrow \infty}\left|a_{k}\right|=0$, the function $f$ belongs to $\mathcal{B}_{0}$ by [27, Theorem 1]. But $\sum_{k=1}^{\infty}\left|a_{k}\right|^{2}=\sum_{k=1}^{\infty} 1 / k=\infty$, and hence $f$ has angular limits almost nowhere on the boundary $\mathbb{T}$ of $\mathbb{D}$ by Zygmund's theorem [31, p. 203]. Further, by the Privalov uniqueness theorem [19, Theorem 10.14], angular limits are non-zero for almost all points where they exist, and it follows that $e^{f}$ has angular limits almost nowhere on $\mathbb{T}$. Thus $e^{f}$ is not of bounded characteristic and therefore $e^{f}$ does not belong to $\bigcup_{p>0} H^{p}$ as claimed.

The remaining part of the paper is organised as follows. Section 2 contains the necessary background material and auxiliary results needed in the proofs of the main results. The proofs themselves can be found in Section 3 where some extension of the main results are also shortly discussed.

\section{BACKGROUND AND AUXILIARY RESULTS}

For $0<s<\infty$, a positive measure $\mu$ on $\mathbb{D}$ is a bounded $s$-Carleson measure, if

$$
\sup _{I} \frac{\mu(S(I))}{|I|^{s}}<\infty
$$

where $|I|$ denotes the arc length of a subarc $I$ of $\mathbb{T}$,

$$
S(I)=\left\{z \in \mathbb{D}: \frac{z}{|z|} \in I, 1-|I| \leqslant|z|\right\}
$$

is the Carleson box based on $I$, and the supremum is taken over all subarcs $I$ of $\mathbb{T}$ such that $|I| \leqslant 1$. Moreover, if

$$
\lim _{|I| \rightarrow 0} \frac{\mu(S(I))}{|I|^{s}}=0
$$


then $\mu$ is a compact (or vanishing) $s$-Carleson measure.

A classical theorem of Carleson $[6,7]$ states that the injection map from the Hardy space $H^{p}$ into the measure space $L^{p}(d \mu)$ is bounded if and only if the positive measure $\mu$ on $\mathbb{D}$ is a bounded Carleson measure. This together with Duren's [11] generalisation is stated as follows.

THEOREM B. Suppose that $\mu$ is a positive measure on $\mathbb{D}$, and let $0<p \leqslant q<\infty$. Then $\mu$ is a bounded $q / p$-Carleson measure if and only if there is a positive constant $C$, depending only on $p$ and $q$, such that

$$
\int_{\mathbb{D}}|f(z)|^{q} d \mu(z) \leqslant C\|f\|_{H^{p}}^{q}
$$

for all analytic functions $f$ in $\mathbb{D}$, in particular for all $f \in H^{p}$. Moreover, if $\mu$ is a bounded $q / p$-Carleson measure, then $C=C_{1} C_{2}$, where $C_{1}>0$ depends only on $p$ and $q$, and

$$
C_{2}=\sup _{I} \frac{\mu(S(I))}{|I|^{q / p}} \text {. }
$$

The following simple lemma is needed while estimating the constant $C$ appearing on the right hand side of (2.3) when the integral on the left hand side is only over some annulus $\mathbb{D} \backslash \Delta(0, t)$. Here and hereafter $\Delta(0, t)$ stands for the Euclidean disc centred at the origin and of radius $t \in(0,1)$.

Lemma 9. Suppose that $\mu$ is a positive measure on $\mathbb{D}$, and let $t \in(0,1)$ and $1 \leqslant s<\infty$. Then

$$
\sup _{I} \frac{\mu(S(I) \backslash \Delta(0, t))}{|I|^{3}} \leqslant 2 \sup _{|I| \leqslant 1-t} \frac{\mu(S(I))}{|I|^{s}} .
$$

Proof: Let $I:=\left\{e^{i \phi}: \theta \leqslant \phi \leqslant \theta+|I|\right\}$ be a subarc of $\mathbb{T}$ such that $|I| \leqslant 1$. If $|I| \leqslant 1-t$ then clearly

$$
\frac{\mu(S(I) \backslash \Delta(0, t))}{|I|^{s}}=\frac{\mu(S(I))}{|I|^{3}} \leqslant \sup _{|I| \leqslant 1-t} \frac{\mu(S(I))}{|I|^{s}} .
$$

If $|I|>1-t$, let $n:=\max \{k \in \mathbb{N}: k(1-t)<|I|\}$, and define $I_{j}:=\left\{e^{i \phi}: \theta+j(1-t)\right.$ $\leqslant \phi \leqslant \theta+(j+1)(1-t)\}$ for $j=0, \ldots, n$. Then $\left|I_{j}\right|=1-t$ for $j=0, \ldots, n$, and therefore

$$
\begin{aligned}
\frac{\mu(S(I) \backslash \Delta(0, t))}{|I|^{s}} & =\frac{1}{|I|^{s}} \int_{S(I)} \chi_{\mathbf{D} \backslash \Delta(0, t)}(z) d \mu(z) \\
& \leqslant \frac{1}{|I|^{s}}\left(\sum_{j=0}^{n-1} \int_{S\left(I_{j}\right)} d \mu(z)+\int_{S\left(I_{n}\right)} \chi_{\mathbb{D} \backslash \Delta(0, t)}(z) d \mu(z)\right) \\
& \leqslant \frac{1}{n^{s}}\left(\sum_{j=0}^{n} \frac{1}{\left|I_{j}\right|^{s}} \int_{S\left(I_{j}\right)} d \mu(z)\right) \\
& \leqslant \frac{n+1}{n^{s}} \sup _{|I| \leqslant 1-t} \frac{\mu(S(I))}{|I|^{s}} \leqslant 2 \sup _{|I|^{s}-t} \frac{\mu(S(I))}{|I|^{s}}
\end{aligned}
$$


The assertion follows by (2.6) and (2.7).

For $a \in \mathbb{D}$, define $\varphi_{a}(z):=(a-z) /(1-\bar{a} z)$, so that $\varphi_{a}$ is the automorphism of $\mathbb{D}$ which interchanges zero and the point $a$. It is known that a positive measure $\mu$ on $\mathbb{D}$ is a bounded $s$-Carleson measure if and only if

$$
\sup _{a \in \mathbb{D}} \int_{\mathbb{D}}\left|\varphi_{a}^{\prime}(z)\right|^{s} d \mu(z)<\infty,
$$

and moreover the supremums in (2.1) and (2.8) are comparable, see [4, 14]. The following partial boundary version of this result will be needed later.

Lemma 10. Suppose that $\mu$ is a positive measure on $\mathbb{D}$, and let $t \in(0,1)$ and $0<s<\infty$. Then

$$
\sup _{|I| \leqslant 1-t} \frac{\mu(S(I))}{|I|^{s}} \leqslant 10^{s} \sup _{|a| \geqslant t} \int_{\mathbb{D} \backslash \Delta(0, t)}\left|\varphi_{a}^{\prime}(z)\right|^{s} d \mu(z) .
$$

Proof: Let $I \subset \mathbb{T}$ such that $|I| \leqslant 1-t$, and let $z \in S(I)$. Choose $a:=(1-|I|) e^{i \theta}$, where $e^{i \theta}$ is the midpoint of $I$, so that every $I$ on $\mathbb{T}$ with $|I| \leqslant 1-t$ corresponds to a unique $a \in \mathbb{D}$ with $|a| \geqslant t$, and vice versa. Since $|1-\bar{a} z| \leqslant 1-|a|^{2}+|z-a|$, and

$$
\begin{aligned}
|z-a|^{2} & \leqslant\left|e^{i(\theta+|I| / 2)}-(1-|I|) e^{i \theta}\right|^{2} \\
& =1-2(1-|I|) \Re\left(e^{-i(I I / 2)}\right)+(1-|I|)^{2} \\
& \leqslant 1-2(1-|I|)\left(1-\frac{|I|^{2}}{8}\right)+(1-|I|)^{2} \leqslant \frac{5}{4}|I|^{2},
\end{aligned}
$$

one obtains $\left|\varphi_{a}^{\prime}(z)\right| \geqslant(10|I|)^{-1}$ for all $z \in S(I)$, and the inequality (2.9) follows.

The following result on radial growth of functions in weighted Bergman spaces can be found in [25].

Lemma C. Suppose that $0<p<\infty,-1<\alpha<\infty$ and $f \in A_{\alpha}^{p}$. Then

$$
|f(z)|\left(1-|z|^{2}\right)^{(2+\alpha) / p} \leqslant\|f\|_{A_{\alpha}^{p}}, \quad z \in \mathbb{D}
$$

with equality only for constant multiples of the function $\left(\varphi_{a}^{\prime}\right)^{(2+\alpha) / p}$.

\section{Proofs and generalisations}

The proof of Theorem 1 is now given in detail. It is constructed such that it does not depend too much on Hilbert space techniques, and therefore the reasoning may be followed mutatis mutandis while proving Theorem 6 . The notation $a \lesssim b$ means that $a \leqslant C b$ for some positive constant $C$, independent of $a$ and $b$, and $a \gtrsim b$ is understood in an analogous manner. 
Proof of Theorem 1: For an analytic function $f(z)=\sum_{k=0}^{\infty} a_{k} z^{k}$ in $\mathbb{D}$, let

$$
T_{n} f(z):=\sum_{k=0}^{n} a_{k} z^{k}, \quad R_{n} f(z):=\sum_{k=n+1}^{\infty} a_{k} z^{k} .
$$

Since $T_{n}$ is compact on $H^{2}$,

$$
\left\|J_{g}\right\|_{e}=\left\|J_{g}\left(T_{n}+R_{n}\right)\right\|_{e} \leqslant\left\|J_{g} T_{n}\right\|_{e}+\left\|J_{g} R_{n}\right\|_{e}=\left\|J_{g} R_{n}\right\|_{e} \leqslant\left\|J_{g} R_{n}\right\|,
$$

and it follows that

$$
\begin{aligned}
\left\|J_{g}\right\|_{e}^{2} & \leqslant \liminf _{n \rightarrow \infty}\left\|J_{g} R_{n}\right\|^{2} \leqslant \liminf _{n \rightarrow \infty} \sup _{\|f\|_{H^{2}} \leqslant 1}\left\|\left(J_{g} R_{n}\right)(f)\right\|_{H^{2}}^{2} \\
& =\liminf _{n \rightarrow \infty} \sup _{\|f\|_{H^{2} \leqslant 1}} 2 \int_{\mathbb{D}}\left|R_{n} f(z)\right|^{2}\left|g^{\prime}(z)\right|^{2} \log \frac{1}{|z|} d A(z) \\
& =\liminf _{n \rightarrow \infty} \sup _{\|f\|_{H^{2}} \leqslant 1} 2 \int_{\mathbb{D} \backslash \Delta(0, r)}\left|R_{n} f(z)\right|^{2}\left|g^{\prime}(z)\right|^{2} \log \frac{1}{|z|} d A(z)
\end{aligned}
$$

for any $0<r<1$, since

$$
\liminf _{n \rightarrow \infty} \sup _{\|f\|_{H^{2}} \leqslant 1} \int_{\Delta(0, r)}\left|R_{n} f(z)\right|^{2}\left|g^{\prime}(z)\right|^{2} \log \frac{1}{|z|} d A(z) \lesssim\left(\max _{|z| \leqslant r}\left|g^{\prime}(z)\right|\right)^{2} \liminf _{n \rightarrow \infty} r^{2(n+1)}=0 .
$$

By Theorem B, and Lemmas 9 and 10,

$$
\begin{aligned}
& \int_{\mathbb{D} \backslash \Delta(0, r)}\left|R_{n} f(z)\right|^{2}\left|g^{\prime}(z)\right|^{2} \log \frac{1}{|z|} d A(z) \\
& \quad \leqslant C \sup _{I} \frac{1}{|I|} \int_{S(I)} \chi \mathbb{D} \backslash \Delta(0, r)(z)\left|g^{\prime}(z)\right|^{2} \log \frac{1}{|z|} d A(z)\left\|R_{n} f\right\|_{H^{2}}^{2} \\
& \leqslant 2 C \sup _{|I| \leqslant 1-r} \frac{1}{|I|} \int_{S(I)}\left|g^{\prime}(z)\right|^{2} \log \frac{1}{|z|} d A(z)\|f\|_{H^{2}}^{2} \\
& \leqslant 20 C \sup _{|a| \geqslant r} \int_{\mathbb{D} \backslash \Delta(0, r)}\left|\varphi_{a}^{\prime}(z)\left\|\left.g^{\prime}(z)\right|^{2} \log \frac{1}{|z|} d A(z)\right\| f \|_{H^{2}}^{2}\right. \\
& \leqslant \frac{20 C}{r} \sup _{|a| \geqslant r} \int_{\mathbb{D}}\left|g^{\prime}(z)\right|^{2}\left(1-\left|\varphi_{a}(z)\right|^{2}\right) d A(z)\|f\|_{H^{2}}^{2},
\end{aligned}
$$

where the fact $\left\|R_{n} f\right\|_{H^{2}} \leqslant\|f\|_{H^{2}}$ and the inequality $-\log |z| \leqslant r^{-1}\left(1-|z|^{2}\right)$ for $r \leqslant|z|<1$ have also been used. As $r \rightarrow 1$ it follows that

$$
\left\|J_{g}\right\|_{e}^{2} \leqslant 40 C \limsup _{|a| \rightarrow 1^{-}} \int_{\mathbf{D}}\left|g^{\prime}(z)\right|^{2}\left(1-\left|\varphi_{\mathbf{a}}(z)\right|^{2}\right) d A(z) .
$$

To obtain the lower bound, choose $f_{a}(z):=\left(\varphi_{a}^{\prime}(z)\right)^{1 / 2}$ for $a \in \mathbb{D}$. Then $\left\|f_{a}\right\|_{H^{2}}=1$, 
and $f_{a} \rightarrow 0$ uniformly on compact subsets of $\mathbb{D}$ as $|a| \rightarrow 1$. If $K$ is compact on $H^{2}$, then

$$
\begin{aligned}
\left\|J_{g}-K\right\|^{2} & \geqslant \limsup _{|a| \rightarrow 1^{-}}\left\|J_{g}\left(f_{a}\right)-K\left(f_{a}\right)\right\|_{H^{2}}^{2} \\
& \geqslant \underset{|a| \rightarrow 1^{-}}{\limsup }\left\|J_{g}\left(f_{a}\right)\right\|_{H^{2}}^{2}-\underset{|a| \rightarrow 1^{-}}{\limsup }\left\|K\left(f_{a}\right)\right\|_{H^{2}}^{2} \\
& =\underset{|a| \rightarrow 1^{-}}{\limsup }\left\|J_{g}\left(f_{a}\right)\right\|_{H^{2}}^{2},
\end{aligned}
$$

and it follows that

$$
\begin{aligned}
\left\|J_{g}\right\|_{e}^{2} & \geqslant \limsup _{|a| \rightarrow 1^{-}} 2 \int_{\mathbb{D}}\left|\varphi_{a}^{\prime}(z)\right|\left|g^{\prime}(z)\right|^{2} \log \frac{1}{|z|} d A(z) \\
& \geqslant \limsup _{|a| \rightarrow 1^{-}} \int_{\mathbb{D}}\left|g^{\prime}(z)\right|^{2}\left(1-\left|\varphi_{a}(z)\right|^{2}\right) d A(z),
\end{aligned}
$$

where the last estimate follows by the inequality $-2 \log |z| \geqslant 1-|z|^{2}$ for $z \in \mathbb{D} \backslash\{0\}$. $\square$

A generalisation of Theorem 1 is now shortly discussed. For $0<p<\infty$, the Dirichlet-type space $D^{p}$ consists of those functions $f \in H(\mathbb{D})$ for which

$$
\|f\|_{D^{p}}:=\left(\int_{\mathbb{D}}\left|f^{\prime}(z)\right|^{p}\left(1-|z|^{2}\right)^{p-1} d A(z)\right)^{1 / p}<\infty .
$$

If $p=2$, then $D^{p}$ is the Hardy space $H^{2}$, and $H^{p} \subset D^{p}$ for $p>2$ by a classical result by Littlewood and Paley $[15,17]$. If $0<p<2$, then $D^{p} \subset H^{p}$ by the following lemma due to Flett, Vinogradov, and $W u[13,24,26]$.

Lemma $\mathrm{D}$. If $f$ is an analytic function in $\mathbb{D}$ and $0<p \leqslant 2$, then $\|f\|_{H^{p}}$ $\lesssim\|f\|_{D^{p}}+|f(0)|$.

For $p>0$, a function $f \in H(\mathbb{D})$ belongs to the Besov-type space $B^{p}$ if

$$
\|f\|_{B^{p}}:=\left(\sup _{a \in \mathbb{D}} \int_{\mathbf{D}}\left|f^{\prime}(z)\right|^{p}\left(1-|z|^{2}\right)^{p-2} g(z, a) d A(z)\right)^{1 / p}<\infty .
$$

Obviously $B^{p}$ is a Möbius invariant subspace of $D^{p}$. The closure of polynomials in $B^{p}$ is denoted by $B_{0}^{p}$, and it consists of those functions $f \in H(\mathbb{D})$ for which the integral in (3.4) tends to zero as $|a| \rightarrow 1$.

PROPOSITION 11. Let $0<p \leqslant 2$. Then $J_{g}$ is bounded on $D^{p}$ if and only if $g \in B^{p}$.

PROOF: The proof is straightforward and therefore it is merely sketched. By Theorem B, Lemma D, and the fact that the supremums in (2.1) and (2.8) are comparable,

$$
\begin{aligned}
\left\|J_{g}(f)\right\|_{D^{p}}^{p} & =\int_{\mathbb{D}}|f(z)|^{p}\left|g^{\prime}(z)\right|^{p}\left(1-|z|^{2}\right)^{p-1} d A(z) \\
& \lesssim \sup _{I} \frac{1}{|I|} \int_{S(I)}\left|g^{\prime}(z)\right|^{p}\left(1-|z|^{2}\right)^{p-1} d A(z)\|f\|_{H^{p}}^{p} \\
& \lesssim \sup _{a \in \mathbb{D}} \int_{\mathbb{D}}\left|g^{\prime}(z)\right|^{p}\left(1-|z|^{2}\right)^{p-2}\left(1-\left|\varphi_{a}(z)\right|^{2}\right) d A(z)\left(\|f\|_{D^{p}}+|f(0)|\right)^{p},
\end{aligned}
$$


and it follows by the inequality $1-\left|\varphi_{a}(z)\right|^{2} \leqslant 2 g(z, a), z \in \mathbb{D} \backslash\{a\}$, that $J_{g}$ is bounded on $D^{p}$ if $g \in B^{p}$. The only if part of the assertion can be deduced by using the test functions $\left(\varphi_{a}^{\prime}(z)\right)^{1 / p}, a \in \mathbb{D}$.

Theorem 1 is the special case $p=2$ of Proposition 12 below.

Proposition 12. Suppose that $1<p \leqslant 2$. If $J_{g}$ is bounded on $D^{p}$ then

$$
\left\|J_{g}\right\|_{e}^{p} \simeq \limsup _{|a| \rightarrow 1^{-}} \int_{\mathbb{D}}\left|g^{\prime}(z)\right|^{p}\left(1-|z|^{2}\right)^{p-2}\left(1-\left|\varphi_{a}(z)\right|^{2}\right) d A(z) .
$$

Proof: The proof is essentially same as the proof of Theorem 1 . The only extra ingredient needed is Lemma 3 , and the appropriate test functions are ones again $\left(\varphi_{a}^{\prime}(z)\right)^{1 / p}$, $a \in \mathbb{D}$. In order to avoid unnecessary repetitions the details are omitted.

Proposition 12 has the following immediate consequence.

Corollary 13. Let $1<p \leqslant 2$. Then $J_{g}$ is compact on $D^{p}$ if and only if $g \in B_{0}^{p}$.

It is now proceeded to prove the rest of the results presented in the introduction. The well-known fact that

$$
\|f\|_{A_{\alpha}^{p}} \simeq\left\|f^{\prime}\right\|_{A_{p+\alpha}^{p}}+|f(0)|
$$

for $f \in H(\mathbb{D})$ is repeatedly used in the subsequent proofs. Since $J_{g}(f)(0)=0$, formula (3.5) gives $\left\|J_{g}(f)\right\|_{A_{\alpha}^{p}} \simeq\left\|\left(J_{g}(f)\right)^{\prime}\right\|_{A_{p+\alpha}^{p}}$ for all $0<p<\infty$ and $-1<\alpha<\infty$.

Proof of Theorem 2: (i) If $f \in A_{\alpha}^{p}$, then by (3.5) and Lemma C,

$$
\begin{aligned}
& \left\|J_{g}(f)\right\|_{A_{\beta}^{q}}^{q} \simeq \int_{\mathbb{D}}|f(z)|^{q}\left|g^{\prime}(z)\right|^{q}\left(1-|z|^{2}\right)^{q+\beta} d A(z) \\
& \leqslant\|g\|_{\mathcal{B}^{1+(2+\beta) / q-(2+\alpha) / p}}^{q} \int_{\mathbb{D}}|f(z)|^{q}\left(1-|z|^{2}\right)^{(q / p)(2+\alpha)-2} d A(z) \\
& =\|g\|_{B^{1+(2+\beta) / q-(2+\alpha) / p}}^{q} \int_{\mathbb{D}}|f(z)|^{p}\left(1-|z|^{2}\right)^{\alpha}|f(z)|^{q-p}\left(1-|z|^{2}\right)^{(q / p)(2+\alpha)-2-\alpha} d A(z) \\
& \leqslant\|g\|_{\mathcal{B}^{1+(2+\beta) / q-(2+\alpha) / p}}^{q}\|f\|_{A_{\alpha}^{p}}^{q-p} \int_{\mathbb{D}}|f(z)|^{p}\left(1-|z|^{2}\right)^{\alpha} d A(z) \\
& \simeq\|g\|_{B^{1+(2+\beta) / q-(2+a) / p}}^{q}\|f\|_{A_{a}^{p}}^{q},
\end{aligned}
$$

and therefore $J_{g}: A_{\alpha}^{p} \rightarrow A_{\beta}^{q}$ is bounded if $g \in \mathcal{B}^{1+(2+\beta) / q-(2+\alpha) / p}$.

Assume now that $J_{g}: A_{\alpha}^{p} \rightarrow A_{\beta}^{q}$ is bounded and let $f_{a}(z):=\left(\varphi_{a}^{\prime}(z)\right)^{(2+\alpha) / p}$. Then, by Lemma 2 and (3.5),

$$
\begin{aligned}
\frac{\left|g^{\prime}(a)\right|}{\left(1-|a|^{2}\right)^{(\alpha+2) / p}} & =\left|\left(J_{g}\left(f_{a}\right)\right)^{\prime}(a)\right| \leqslant \frac{\left\|J_{g}\left(f_{a}\right)^{\prime}\right\|_{A_{q+\beta}^{q}}}{\left(1-|a|^{2}\right)^{(q+\beta+2) / q}} \simeq \frac{\left\|J_{g}\left(f_{a}\right)\right\|_{A_{\beta}^{q}}}{\left(1-|a|^{2}\right)^{1+(2+\beta) / q}} \\
& \lesssim \frac{\left\|f_{a}\right\|_{A_{\alpha}^{p}}}{\left(1-|a|^{2}\right)^{1+(2+\beta) / q}}=\frac{1}{\left(1-|a|^{2}\right)^{1+(2+\beta) / q}}
\end{aligned}
$$


and it follows that $g \in \mathcal{B}^{1+(2+\beta) / q-(2+\alpha) / p}$.

(ii) By the Hölder inequality,

$$
\begin{aligned}
\left\|T_{g}(f)\right\|_{A_{\beta}^{q}}^{q} & \simeq \int_{\mathbb{D}}|f(z)|^{q}\left|g^{\prime}(z)\right|^{q}\left(1-|z|^{2}\right)^{q+\beta} d A(z) \\
& =\int_{\mathbb{D}}|f(z)|^{q}\left(1-|z|^{2}\right)^{(\alpha q) / p}\left|g^{\prime}(z)\right|^{q}\left(1-|z|^{2}\right)^{q+\beta-(\alpha q) / p} d A(z) \\
\leqslant & \left(\int_{\mathbb{D}}|f(z)|^{p}\left(1-|z|^{2}\right)^{\alpha} d A(z)\right)^{q / p} \\
& \quad\left(\int_{\mathbb{D}}\left|g^{\prime}(z)\right|^{p / q} p-q\left(1-|z|^{2}\right)^{p /(p-q)(q+\beta-(\alpha q) / p)} d A(z)\right)^{(q-p) / p},
\end{aligned}
$$

and since $1 / s=1 / q-1 / p$, that is, $s=(p q) /(p-q)$, and

$$
\frac{p}{p-q}\left(q+\beta-\frac{\alpha q}{p}\right)=\frac{p \beta-\alpha q}{p-q}+\frac{p q}{p-q},
$$

it follows that $J_{g}: A_{\alpha}^{p} \rightarrow A_{\beta}^{q}$ is bounded if $g^{\prime} \in A_{s+\gamma}^{s}$, where $\gamma=(p \beta-\alpha q) /(p-q)$.

If $J_{g}: A_{\alpha}^{p} \rightarrow A_{\beta}^{q}$ is bounded, then a generalisation $[16,5.1]$ of a result by Luecking $[18$, Theorem 1] implies

$$
C(g):=\int_{\mathbb{D}}\left(\frac{\int_{D(z, 1 / 2)}\left|g^{\prime}(w)\right|^{q}\left(1-|w|^{2}\right)^{q+\beta} d A(w)}{\left(1-|z|^{2}\right)^{2+\alpha}}\right)^{p /(p-q)} d A(z)<\infty,
$$

where $D(z, 1 / 2):=\left\{w:\left|\varphi_{z}(w)\right|<1 / 2\right\}$ is the pseudohyperbolic disc of centre $z$ and radius $1 / 2$. But by the subharmonicity,

$$
\left|g^{\prime}(z)\right|^{q} \lesssim \frac{\int_{D(z, 1 / 2)}\left|g^{\prime}(w)\right|^{q} d A(w)}{\left(1-|z|^{2}\right)^{2}},
$$

and since $1-|z|^{2} \simeq 1-|w|^{2}$ for $w \in D(z, 1 / 2)$, it follows that $\left\|g^{\prime}\right\|_{A_{i+\gamma}^{s}}^{s_{i}} \lesssim C(g)$, where $1 / s=1 / q-1 / p$ and $\gamma=(p \beta-\alpha q) /(p-q)$.

(iii) The assertion follows by (3.6).

Proof of Theorem 5: (i) If $f \in H^{p}$, then by Theorem B and the fact that the supremums in (2.1) and (2.8) are comparable,

$$
\begin{aligned}
\left\|T_{g}(f)\right\|_{A_{\beta}^{q}}^{q} & \simeq \int_{\mathbb{D}}|f(z)|^{q}\left|g^{\prime}(z)\right|^{q}\left(1-|z|^{2}\right)^{q+\beta} d A(z) \\
& \lesssim \sup _{a \in \mathbb{D}} \int_{\mathbb{D}}\left|\varphi_{a}^{\prime}(z)\right|^{(q / p)}\left|g^{\prime}(z)\right|^{q}\left(1-|z|^{2}\right)^{q+\beta} d A(z)\|f\|_{H^{p}}^{q} \\
& \leqslant\|g\|_{\mathcal{B}^{1+(2+\beta) / q-1 / p}}^{q}\|f\|_{H^{p}}^{q} \sup _{a \in \mathbb{D}} \int_{\mathbf{D}} \frac{\left(1-|a|^{2}\right)^{q / p}\left(1-|z|^{2}\right)^{q / p-2}}{|1-\bar{a} z|^{2(q / p)}} d A(z),
\end{aligned}
$$


and since the integral above is uniformly bounded by Forelli-Rudin estimates [30, Lemma 4.2.2], it follows that $J_{g}: H^{p} \rightarrow A_{\beta}^{q}$ is bounded if $g \in \mathcal{B}^{1+(2+\beta) / q-1 / p}$.

Assume now that $J_{g}: H^{p} \rightarrow A_{\beta}^{q}$ is bounded and let $f_{a}(z):=\left(\varphi_{a}^{\prime}(z)\right)^{1 / p}$. Then the reasoning in (3.6) remains valid for $\alpha=-1$, and it follows that $g \in \mathcal{B}^{1+(2+\beta) / q-1 / p}$.

(ii) If $f \in H^{p}$, then, by Theorem B and the fact that the supremums in (2.1) and (2.8) are comparable,

$$
\begin{aligned}
\left\|T_{g}(f)\right\|_{A_{\boldsymbol{\beta}}^{p}}^{p} & \simeq \int_{\mathbb{D}}|f(z)|^{p}\left|g^{\prime}(z)\right|^{p}\left(1-|z|^{2}\right)^{p+\beta} d A(z) \\
& \lesssim \sup _{a \in \mathbb{D}} \int_{\mathbb{D}}\left|\varphi_{a}^{\prime}(z)\left\|\left.g^{\prime}(z)\right|^{p}\left(1-|z|^{2}\right)^{p+\beta} d A(z)\right\| f \|_{H^{p}}^{p}\right. \\
& =\sup _{a \in \mathbb{D}} \int_{\mathbb{D}}\left|g^{\prime}(z)\right|^{p}\left(1-|z|^{2}\right)^{p+\beta-1}\left(1-\left|\varphi_{a}(z)\right|^{2}\right) d A(z)\|f\|_{H^{p}}^{p}
\end{aligned}
$$

and therefore $J_{g}: H^{p} \rightarrow A_{\beta}^{p}$ is bounded if (1.2) is satisfied.

Assume now that $J_{g}: H^{p} \rightarrow A_{\beta}^{p}$ is bounded and let $f_{a}(z):=\left(\varphi_{a}^{\prime}(z)\right)^{1 / p}$. Then

$$
\sup _{a \in \mathbb{D}} \int_{\mathbb{D}}\left|\varphi_{a}^{\prime}(z)\right|\left|g^{\prime}(z)\right|^{p}\left(1-|z|^{2}\right)^{p+\beta} d A(z) \lesssim \sup _{a \in \mathbb{D}}\left\|f_{a}\right\|_{H^{p}}^{p}=1,
$$

and (1.2) follows.

(iii) The assertion follows by (3.6) since the reasoning remains valid for $\alpha=-1$. 0

Proof of Theorem 6: (i) Denote

$$
C(g):=\limsup _{|z| \rightarrow 1^{-}}\left|g^{\prime}(z)\right|\left(1-|z|^{2}\right)^{1+(2+\beta) / q-(2+\alpha) / p},
$$

so that there is an $r_{\varepsilon} \in(0,1)$ such that

$$
\left|g^{\prime}(z)\right|\left(1-|z|^{2}\right)^{1+(2+\beta) / q-(2+\alpha) / p} \leqslant C(g)+\varepsilon
$$

for all $|z| \geqslant r_{\varepsilon}$. Let the operators $T_{n}$ and $R_{n}$ be defined as in the proof of Theorem 1 . Then $T_{n}$ is compact on $A_{\alpha}^{p}$, and the reasoning in (3.1) and (3.2) yields

$$
\begin{aligned}
\left\|J_{g}\right\|_{e}^{q} & \lesssim \liminf _{n \rightarrow \infty} \sup _{\|f\|_{A_{\alpha}^{p} \leqslant 1}} \int_{\mathbb{D} \backslash \Delta\left(0, r_{e}\right)}\left|R_{n} f(z)\right|^{q}\left|g^{\prime}(z)\right|^{q}\left(1-|z|^{2}\right)^{q+\beta} d A(z) \\
& \leqslant(C(g)+\varepsilon)^{q} \liminf _{n \rightarrow \infty} \sup _{\|f\|_{A_{a}^{p} \leqslant 1}} \int_{\mathbb{D}}\left|R_{n} f(z)\right|^{q}\left(1-|z|^{2}\right)^{((2+a) q / p)-2} d A(z) .
\end{aligned}
$$

But now

$$
\int_{\mathbb{D}}\left|R_{n} f(z)\right|^{q}\left(1-|z|^{2}\right)^{((2+\alpha) q / p)-2} d A(z) \lesssim\left\|R_{n} f\right\|_{A_{\alpha}^{q}}^{q}
$$

by Lemma 2 , and since $\left\|R_{n} f\right\|_{A_{a}^{p}} \lesssim\|f\|_{A_{a}^{p}}$ it follows that $\left\|J_{g}\right\|_{e} \lesssim C(g)$. 
To obtain the lower bound, choose $f_{a}(z):=\left(\varphi_{a}^{\prime}(z)\right)^{(2+a) / p}$ for $a \in \mathbb{D}$. Then $\left\|f_{a}\right\|_{A_{a}^{p}}$ $=1$, and $f_{a} \rightarrow 0$ uniformly on compact subsets of $\mathbb{D}$ as $|a| \rightarrow 1$. A similar reasoning as in (3.3) shows that

$$
\left\|J_{g}\right\|_{e}^{q} \gtrsim \limsup _{|a| \rightarrow 1^{-}} \int_{\mathbb{D}}\left|g^{\prime}(z)\right|^{q}\left(1-|z|^{2}\right)^{q+\beta-(q(2+\alpha) / p)}\left(1-\left|\varphi_{a}(z)\right|^{2}\right)^{(q(2+\alpha)) / p} d A(z) .
$$

By the subharmonicity of $g^{\prime} \circ \varphi_{a}$,

$$
\left|g^{\prime}(a)\right|^{q} \leqslant \frac{64}{\pi\left(1-|a|^{2}\right)^{2}} \int_{D(a, 1 / 2)}\left|g^{\prime}(z)\right|^{q} d A(z),
$$

and since $1-|a|^{2} \simeq 1-|z|^{2} \simeq|1-\bar{a} z|$ for $z \in D(a, 1 / 2)$, this yields

$$
\begin{aligned}
\left(\left|g^{\prime}(a)\right|\left(1-|a|^{2}\right)^{1+(2+\beta) / q-(2+\alpha) / p}\right)^{q} & \\
& \lesssim \int_{\mathbb{D}}\left|g^{\prime}(z)\right|^{q}\left(1-|z|^{2}\right)^{q+\beta-(q(2+\alpha)) / p}\left(1-\left|\varphi_{a}(z)\right|^{2}\right)^{(q(2+\alpha)) / p} d A(z) .
\end{aligned}
$$

It follows that $C(g) \lesssim\left\|J_{g}\right\|_{e}$, and the proof of (i) is complete.

(ii) Denote by $C(q)$ the limit superior in (3.7) with $\alpha=-1$, so that (3.8) with $\alpha=-1$ is satisfied for all $|z| \geqslant r_{\varepsilon}$. The reasoning in (3.1) and (3.2) gives

$$
\left\|J_{g}\right\|_{e}^{q} \lesssim \liminf _{n \rightarrow \infty} \sup _{\|f\|_{H^{p}} \leqslant 1} \int_{\mathbb{D} \backslash \Delta\left(0, r_{e}\right)}\left|R_{n} f(z)\right|^{q}\left|g^{\prime}(z)\right|^{q}\left(1-|z|^{2}\right)^{q+\beta} d A(z)
$$

from which Theorem B and Lemmas 9 and 10 yield

$$
\begin{aligned}
\int_{\mathbb{D} \backslash \Delta\left(0, r_{\varepsilon}\right)} & \left|R_{n} f(z)\right|^{q}\left|g^{\prime}(z)\right|^{q}\left(1-|z|^{2}\right)^{q+\beta} d A(z) \\
& \lesssim \sup _{a \in \mathbb{D}} \int_{\mathbb{D} \backslash \Delta\left(0, r_{\varepsilon}\right)}\left|\varphi_{a}^{\prime}(z)\right|^{q / p}\left|g^{\prime}(z)\right|^{q}\left(1-|z|^{2}\right)^{q+\beta} d A(z)\left\|R_{n} f\right\|_{H^{p}}^{q} \\
& \leqslant(C(g)+\varepsilon)^{q}\left\|R_{n} f\right\|_{H^{p}}^{q} \sup _{a \in \mathbb{D}} \int_{\mathbb{D}} \frac{\left(1-|a|^{2}\right)^{q / p}\left(1-|z|^{2}\right)^{(q / p)-2}}{|1-\bar{a} z|^{2(q / p)}} d A(z) .
\end{aligned}
$$

Since the integral above is uniformly bounded by Forelli-Rudin estimates [29, Lemma 4.2.2], it follows that $\left\|J_{g}\right\|_{e} \lesssim C(g)$.

The lower bound can be obtained by using the functions $f_{a}(z):=\left(\varphi_{a}^{\prime}(z)\right)^{1 / p}$ and following the second part of the proof of Case (i).

(iii) The upper bound follows by applying Theorem $B$ and Lemmas 9 and 10, and the lower bound can be obtained by using the functions $f_{a}(z):=\left(\varphi_{a}^{\prime}(z)\right)^{1 / p}$. The details are omitted.

\section{REFERENCES}

[1] A. Aleman and J.A. Cima, 'An integral operator on $H^{p}$ and Hardy's inequality', J. Anal. Math. 85 (2001), 157-176. 
[2] A. Aleman and A.G. Siskakis, 'An integral operator on $H^{p}$ ', Complex Variables Theory Appl. 28 (1995), 149-158.

[3] A. Aleman and A.G. Siskakis, 'Integration operators on Bergman spaces', Indiana Univ. Math. J. 46 (1997), 337-356.

[4] R. Aulaskari, D. Stegenga, and J. Xiao, 'Some subclasses of $B M O A$ and their characterization in terms of Carleson measures', Rocky Mountain J. Math. 26 (1996), 485-506.

[5] A. Baernstein II, 'Analytic functions of bounded mean oscillation', in Aspects of Contemporary Complex Analysis, (D. Brannan and J. Clunie, Editors) (Academic Press, London, New York, 1980), pp. 3-36.

[6] L. Carleson, 'An interpolation problem for bounded analytic functions', Amer. J. Math. 80 (1958), 921-930.

[7] L. Carleson, 'Interpolations by bounded analytic functions and the corona problem', Ann. of Math. (2) 76 (1962), 547-559.

[8] J.A. Cima and G. Schober, 'Analytic functions with bounded mean oscillation and logarithms of $H^{p}$ functions', Math. $Z .151$ (1976), 295-300.

[9] W.S. Cohn, 'A factorization theorem for the derivative of a function in $H^{p}$, Proc. Amer. Math. Soc. 127 (1999), 509-517.

[10] C.C. Cowen and B.D. MacCluer, Composition operators on spaces of analytic functions, Studies in Advanced Mathematics (CRC Press, Boca Raton, FL, 1995).

[11] P. Duren, 'Extension of a Theorem of Carleson', Bull. Amer. Math. Soc. 75 (1969), 143-146.

[12] P. Duren, Theory of $H^{p}$ spaces (Academic Press, New York, London, 1970).

[13] T.M. Flett, 'The dual of an inequality of Hardy and Littlewood and some related inequalitites', J. Math. Anal. Appl. 38 (1972), 746-765.

[14] J. Garnett, Bounded analytic functions (Academic Press, New York, 1981).

[15] G.H. Hardy and J.E. Littlewood, 'Some more theorems concerning Fourier series and Fourier power series', Duke Math. J. 2 (1936), 354-382.

[16] H. Jarchow, 'Embeddings induced by planar Carleson measures', in Function spaces and operator theory, Proceedings of the Summer School held at the University of Joensuu, Joensur, May 19-23, 2003, (Jari Taskinen, Editor), University of Joensuu, Department of Mathematics. Report Series, 8 (University of Joensuu, Joensuu, 2005).

[17] D.H. Luecking, 'A new proof of an inequality of Littlewood and Paley', Proc. Amer. Math. Soc. 103 (1988), 887-893.

[18] D.H. Luecking, 'Embedding theorems for spaces of analytic functions via Khinchine's inequality', Michigan Math. J. 40 (1993), 333-358.

[19] Ch. Pommerenke, Univalent functions, Studia Mathematica/Mathematische Lehrbücher, Band XXV, (With a chapter on quadratic differentials by Gerd Jensen (Vandenhoeck and Ruprecht, Göttingen, 1975).

[20] Ch. Pommerenke, 'Schlichte Funktionen und analytische Funktionen von beschränkter mittlerer Oszillation', (German), Comment. Math. Helv. 52 (1977), 591-602.

[21] J.H. Shapiro, 'The essential norm of a composition operator', Ann. Math. 125 (1987), 375-404.

[22] A.G. Siskakis, Studies on composition operators (Laramie, WY, 1996), in, Contemp. Math. 213 (Amer. Math. Soc., Providence, RI, 1998), pp. 229-252. 
[23] A.G. Siskakis and R. Zhao, 'A Volterra type operator on spaces of analytic functions', in Function spaces (Edwardsville, IL, 1998), Contemp. Math. 232 (Amer. Math. Soc., Providence, RI, 1999), pp. 299-311.

[24] S.A. Vinogradov, 'Multiplication and division in the space of analytic functions with an area-integrable derivative, and in some related spaces', Issled. po Linein. Oper. i Teor. Funktsii. 23 (1995), 45-77. Translation in J. Math. Sci. (New York) 87 (1997), 3806-3827.

[25] D. Vukotić, 'A sharp estimate for $A_{\alpha}^{p}$ functions in $\mathbb{C}^{n}$, Proc. Amer. Math. Soc. 117 (1993), 753-756.

[26] Z. Wu, 'Carleson measures and multipliers for Dirichlet spaces', J. Func. Anal. 169 (1999), 148-163.

[27] S. Yamashita, 'Gap series and $\alpha$-Bloch functions', Yokohama Math. J. 28 (1980), 31-36.

[28] R. Zhao, 'Pointwise multipliers from weighted Bergman spaces and Hardy spaves on weighted Bergman spaces', Ann. Acad. Sci. Fenn. Math. 29 (2004), 139-150.

[29] K. Zhu, Operator theory in function spaces (Marcel Dekker, New York, 1990).

[30] K. Zhu, 'Translating inequalitites between Hardy and Bergman spaces', Amer. Math. Montly 111 (2004), 520-525.

[31] A. Zygmund, Trigonometric series (Cambridge University Press, London, 1959).

University of Joensuu

Department of Mathematics

P.O. Box 111

80101 Joensuu

Finland

e-mail: jouni.rattya@joensuu.fi 